\title{
Biomedical mass spectrometry
}

\author{
Mitsutoshi Setou $\cdot$ Hisao Oka
}

Published online: 1 April 2011

(C) Springer-Verlag 2011

Nowadays mass spectrometry is increasingly applicable to biomedicine. For many decades, mass spectrometry (MS) has been a major tool for structural analytical chemistry in biomedical fields such as forensic medicine, laboratory medicine, biochemistry, and pharmacology. More recently, after the genome era, proteomics and metabolomics are becoming more popular in the many fields of biology and medicine. In those "omics" approach, mass spectrometric techniques are not only useful but also nearly essential. Recent advances of mass spectrometry are serving the expectation of comprehensive coverage for those "systems biology approaches". In this special issue on biomedical mass spectrometry, stateof-the-art examples of these efforts are published. Some are novel techniques and findings for diagnosis of metabolic diseases by liquid chromatography-MS, electrospray-MS-MS, or gas chromatography-MS. Further, several works are based on imaging MS, which is a brand new approach of MS with spatial information. We

Published in the special issue Biomedical Mass Spectrometry with Guest Editors Hisao Oka and Mitsutoshi Setou.

M. Setou $(\bowtie)$

Department of Molecular Anatomy, Hamamatsu University

School of Medicine,

1-20-1 Handayama,

Hamamatsu, Shizuoka 431-3192, Japan

e-mail: setou@hama-med.ac.jp

\section{H. Oka}

Department of Analytical Chemistry, School of Pharmacy,

Kinjo Gakuin University,

Omori 2-1723, Moriyama-ku,

Nagoya 463-8521, Japan hope readers will benefit from this overview of recent advances in biomedical mass spectrometry.

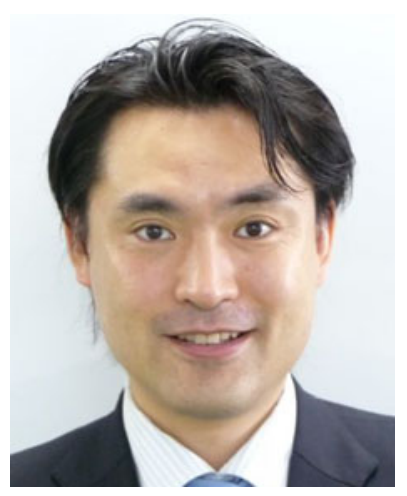

Mitsutoshi Setou has been full professor of Anatomy and Cell Biology at the Hamamatsu University School of Medicine since 2008. His research interests include development and application of imaging mass spectrometry and systems biology with omics technologies. Dr Setou is the author of over 100 peer-reviewed papers, 150 scientific papers, 10 book chapters, and editor of a book on imaging mass spectrometry.

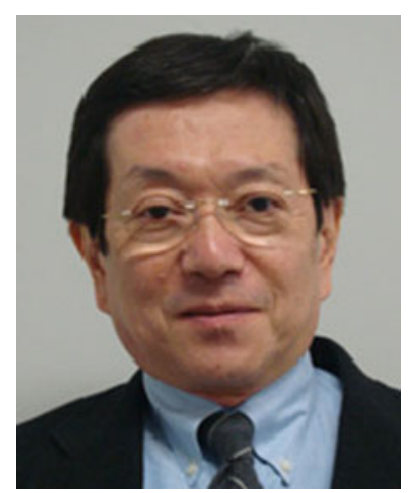

Hisao Oka has been full professor of analytical chemistry at the Kinjo Gakuin University School of Pharmacy since 2005. His research interests include identification and determination of bioactive compounds by liquid chromatography-mass spectrometry. Dr Oka is the author of over 150 peer-reviewed papers and 30 book chapters, and editor of a book on food safety and applications of liquid chromatographymass spectrometry. 